Document downloaded from:

http://hdl.handle.net/10251/121427

This paper must be cited as:

Braunmann, C.; Cortés, J.; Jódar Sánchez, LA.; Villafuerte, L. (2018). On the random Gamma function: theory and computing. Journal of Computational and Applied Mathematics. 335:142-155. https://doi.org/10.1016/j.cam.2017.11.045

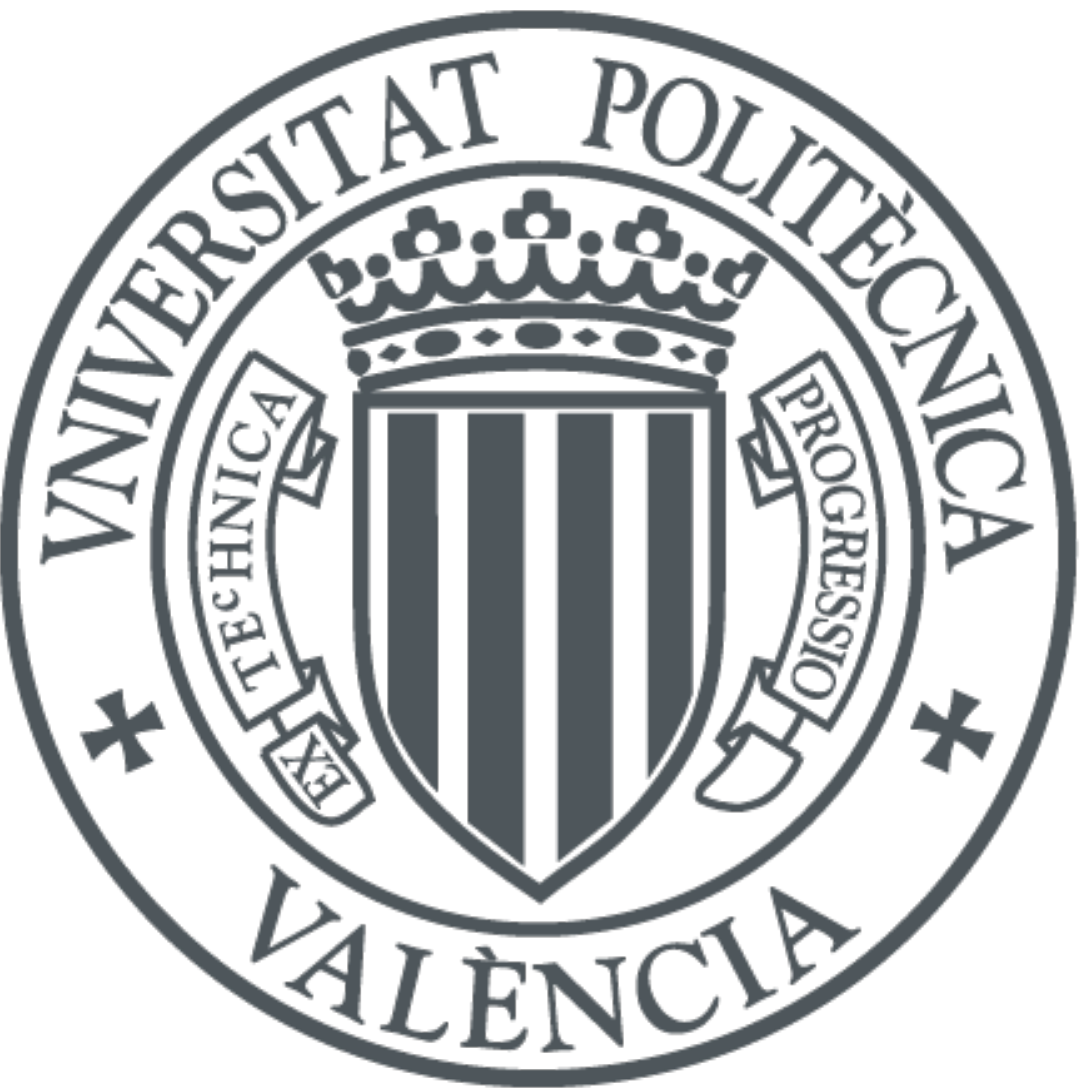

The final publication is available at

http://doi.org/10.1016/j.cam.2017.11.045

Copyright Elsevier

Additional Information 


\title{
On the random Gamma function: theory and computing
}

\author{
C.A. Braumann ${ }^{\mathrm{a}}$, J.-C. Cortés ${ }^{\mathrm{b}}$, L. Jódar ${ }^{\mathrm{b}}$, L. Villafuerte ${ }^{\mathrm{c}, *}$ \\ ${ }^{a}$ Centro de Investigação em Matemática e Aplicações, Instituto de Investigação e \\ Formação Avançada, Universidade de Évora \\ Departamento de Matemática, Escola de Ciências e Tecnologia, Universidade de Évora, \\ Rua Romão Ramalho 59, 7000-671, Évora, Portugal \\ ${ }^{b}$ Instituto Universitario de Matemática Multidisciplinar, Building $8 G 2^{\text {nd }}$ floor Access $C$, \\ Universitat Politècnica de València, 46022 Valencia (Spain) \\ ${ }^{c}$ Department of Mathematics, University of Texas at Austin, USA
}

\section{Abstract}

This paper deals with the extension, in the mean square sense, of the deterministic gamma function to the random framework. In a first step, we provide such extension to $\Gamma(A)$ by assuming that the parameter $A$ is a positive random variable satisfying certain conditions related to its exponential moments. As a particular case, we show that every positive random variable satisfies such conditions if it is bounded and bounded away from zero. In a second step, we establish the formula $\Gamma(A+1)=A \Gamma(A)$ that allows us to extend the random gamma function to a class of random variables whose supports lie over the real line with the exception of small neighborhoods of zero and of the negative integers. This retains the classical definition of the gamma function when $A$ becomes a deterministic parameter. The study is based on the $\mathrm{L}^{p}$ stochastic calculus with $p=2$ and 4 , usually referred to as mean square and mean fourth stochastic calculus, respectively. Next, we compute the mean and the variance of the random gamma function, including several illustrative examples. Finally, with the aid of the random gamma function, we define the random Bessel function and compute reliable approximations of its mean and variance.

Keywords: Random gamma function, mean square stochastic calculus, mean fourth stochastic calculus, stochastic computations.

\footnotetext{
${ }^{*}$ Corresponding Author. Phone number: +34-963-877000-ext.88289. Fax: +34-963877669

Email addresses: braumann@uevora.pt (C.A. Braumann), jccortes@imm.upv.es (J.-C. Cortés), 1jodar@imm.upv.es (L. Jódar), 1va5@hotmail.com, altuzar@math. utexas.edu (L. Villafuerte)
} 


\section{Introduction and motivation}

In its basic definition, the real Euler gamma function is introduced by the following Riemann integral:

$$
\begin{aligned}
\Gamma:] 0, \infty[\subset \mathbb{R} & \rightarrow \mathbb{R} \\
\alpha & \mapsto \int_{0^{+}}^{\infty} e^{-t} t^{\alpha-1} \mathrm{~d} t .
\end{aligned}
$$

This parametric integral is absolutely convergent. This allows the definition of the real Euler gamma function as [1]:

$$
\Gamma(\alpha)=\int_{0^{+}}^{\infty} e^{-t} t^{\alpha-1} \mathrm{~d} t, \quad \alpha>0 .
$$

The Euler gamma function $\Gamma(\alpha)$ appears in describing the solution of some significant deterministic ordinary differential equations (d.o.d.e.'s) whose coefficients and/or initial conditions depend on the parameter $\alpha>0$, such as the Bessel d.o.d.e. [2]. D.o.d.e.'s play an important role in modeling many real problems. In practice, once the continuous model (d.o.d.e.) is selected, setting its inputs (coefficient, source term, initial/boundary conditions) is required. Often this task can only be achieved after measuring certain magnitudes that could not be known exactly due to measuring errors and/or inherent complexity of the phenomenon under study. This uncertainty is transferred to the inputs of the d.o.d.e., such as the parameter $\alpha>0$. As a consequence, in this type of situations it is more realistic to assume that $\alpha$ is a random variable rather than a deterministic constant. This approach motivates the extension of the gamma function to the random framework. Notice that it entails the convergence analysis of the integral (1) which, from a probabilistic stand-point, can be performed in several ways depending on the chosen probabilistic convergence. Throughout this paper, mean square convergence will be used. This is a strong-type of stochastic convergence, so its consideration is advantageous since the results established in the mean square sense entail straightforwardly their extension to other types of stochastic convergence such as convergence in probability and in distribution. At this point it is important to point out that mean square convergence possesses key properties that make it particularly suited for computing the mean and the variance of the random gamma function. These distinctive properties will be apparent later. This is why another types of strong convergence, such 
as the almost sure convergence, have been discarded to conduct our subsequent study. Additionally, we highlight that mean square calculus has been previously applied to extend important deterministic results to the random framework, as can be seen, for instance, in $[3,4,5,6,7,8]$.

Apart from the above arguments, we can add that the random gamma function could be also motivated in the context of fractional differential equations since it is well-know this special function plays a key role in this novel theory [9]. Indeed, in applications the derivative order of fractional differential equations must be determined from sample data. As sampled data usually involve measurement errors, it is natural to consider the derivative order as a random variable instead of a deterministic quantity. This approach also motivates the introduction of random gamma function in order to rigorously study random fractional differential equations in the mean square sense.

To the best of our knowledge, this manuscript is the first to extend the gamma function to the random framework using the mean square convergence. This contribution could then help to generalize other important special functions to the random context in subsequent contributions. To motivate this issue, in this paper we will take advantage of a previous contribution by some of the authors [10], where the random Bessel differential equation has been studied in the mean square sense, to introduce the random Bessel function in a particular scenario (see Example 12). As a consequence, this generalization would enable the mean square study of important randomized differential equations whose solutions, in the classical framework, are represented in terms of special functions.

The paper is organized as follows. In Section 2, the main results concerning $\mathrm{L}^{p}$ stochastic calculus are summarized, particularly for $p=2$ (mean square calculus) and $p=4$ (mean fourth calculus). In Section 3, we introduce the random gamma function, in the m.s. sense, for a class of positive r.v.'s satisfying some exponential moment conditions, which include as a particular case the positive r.v.'s that are bounded and bounded away from zero. This definition is extended to a more general class of real-valued random variables in Section 4, by establishing the classical formula $\Gamma(A+1)=A \Gamma(A)$ in the random scenario and taking advantage of it. Section 5 includes the expressions of the main statistical properties of the random gamma function, namely the expectation and variance, as well as three illustrative examples. Also, the mean and the variance of random Bessel function are computed in a particular case. Conclusions are drawn in the closing section. 


\section{Preliminaries about $\mathrm{L}^{p}$ calculus}

For the sake of clarity, we summarize the main definitions and results that will be used throughout this paper. Further details can be found in [11, ch.1], [12, ch.XI], [13, ch.4], [14, ch.III] and [15].

A real random variable (r.v.) $X: \Omega \rightarrow \mathbb{R}$ defined on a probability space $(\Omega, \mathfrak{F}, \mathbb{P})$ is said to be of order $p \geq 1$ (in short p-r.v.) if $\mathbb{E}\left[|X|^{p}\right]<+\infty$, where $\mathbb{E}[\cdot]$ denotes the expectation operator. The space $L^{p}=L^{p}(\Omega, \mathfrak{F}, \mathbb{P})$ of all $p$-r.v.'s (assuming we do not distinguish between r.v.'s that are equal with probability one), endowed with the norm

$$
\|X\|_{p}=\left(\mathbb{E}\left[|X|^{p}\right]\right)^{1 / p},
$$

is a Banach space $\left[11\right.$, p.9]. The convergence in $\mathrm{L}^{p}$, usually called convergence in $p$-th mean, is the one inferred by the $p$-norm (2).

More generally, let $\mathcal{T}$ denote an arbitrary non-empty index set, a family $X(t) \equiv\{X(t): t \in \mathcal{T}\}$ of r.v.'s is called a stochastic process (s.p.). Throughout this paper, $\mathcal{T}=\left[0, \infty\left[\right.\right.$. If $\mathbb{E}\left[|X(t)|^{p}\right]<+\infty$ for each $t \in \mathcal{T}$, then $\{X(t): t \in \mathcal{T}\}$ is said to be a $p$-stochastic process (p-s.p.). The concepts of $p$-continuity, $p$-differentiability and $p$-integrability of $p$-s.p.'s in $L^{p}$-spaces are the usual ones derived from the $p$-norm in Banach spaces. For instance,

Definition 1. A p-s.p. $\left\{\frac{d X(t)}{d t}: t \in \mathcal{T}\right\}$ is said to be the $p$-derivative of the p-s.p. $\{X(t): t \in \mathcal{T}\}$ with respect to $t$ if

$$
\lim _{\Delta t \rightarrow 0}\left\|\frac{X(t+\Delta t)-X(t)}{\Delta t}-\frac{d X(t)}{d t}\right\|_{p}=0, \quad t, t+\Delta t \in \mathcal{T} .
$$

We illustrate the above definition by means of the following example that will be used later.

Example 1. Let $A \in \mathrm{L}^{4}$. Then $\{X(t)=A \ln (t): t>0\}$ is a 4-s.p. and its 4-derivative with respect to $t$ is given by $\left\{\frac{A}{t}: t>0\right\}$. In fact, first notice that: $\|A \ln (t)\|_{4}=|\ln (t)|\|A\|_{4}<\infty$ for every $t>0$ since $A$ is assumed to be a 4-r.v. Moreover,

$\lim _{\Delta t \rightarrow 0}\left\|\frac{A \ln (t+\Delta t)-A \ln (t)}{\Delta t}-\frac{A}{t}\right\|_{4}=\|A\|_{4} \lim _{\Delta t \rightarrow 0}\left|\frac{\ln (t+\Delta t)-\ln (t)}{\Delta t}-\frac{1}{t}\right|=0$,

where in the last step we have used that $\|A\|_{4}<\infty$ and $(\ln (t))^{\prime}=1 / t$, where $'$ denotes the classical derivative. 
In the particular case that $p=2,\left(\mathrm{~L}^{2},\|\cdot\|_{2}\right)$ is a Hilbert space with the inner product

$$
\langle X, Y\rangle=\mathbb{E}[X Y], \quad X, Y \in \mathrm{L}^{2} .
$$

The convergence associated to the 2-norm inferred by this inner product, which is given by (2) with $p=2$, is usually referred to as mean square (m.s.) convergence. Let $\left\{X_{n}: n \geq 0\right\}$ be a sequence in $\mathrm{L}^{2}$ m.s. convergent to $X \in$ $\mathrm{L}^{2}$ as $n \rightarrow \infty$; in the sequel this will be denoted by $X_{n} \underset{n \rightarrow \infty}{\stackrel{\text { m.s. }}{\longrightarrow}} X$ or $\underset{n \rightarrow \infty}{\lim .} X_{n}=$ $X$, indistinctly. In accordance with previous definitions, the r.v. $\stackrel{n \rightarrow \infty}{X}$ and the s.p. $\{X(t): t>0\}$ in $\mathrm{L}^{2}$ are called 2-r.v.'s and 2-s.p.'s, respectively. For each $t, s>0$, we will for convenience call $\Gamma_{X}(t, s)=\mathbb{E}[X(t) X(s)]$ the covariance function associated to the s.p. $\{X(t): t>0\}$. We point out that many m.s. properties, such as 2-continuity, 2-differentiability, 2-integrability, can be directly characterized through this two-variable deterministic function $[13$, ch.4].

An important result in dealing with $p$-r.v.'s is the Hölder inequality

$$
\mathbb{E}[|X Y|] \leq\|X\|_{p}\|Y\|_{q}, \quad p, q>1, \frac{1}{p}+\frac{1}{q}=1, \quad X \in \mathrm{L}^{p}, Y \in \mathrm{L}^{q} .
$$

Taking $p=q=2$ in (3), one obtains the Schwarz inequality:

$$
\mathbb{E}[|X Y|] \leq\|X\|_{2}\|Y\|_{2}, \quad X, Y \in \mathrm{L}^{2} .
$$

This inequality guarantees that the covariance function $\Gamma_{X}(t, s)$ is well-defined for every 2-s.p.

The following inequality will be crucial in the subsequent development. It deduces directly from the application of the Schwarz inequality:

$$
\|X Y\|_{p} \leq\|X\|_{2 p}\|Y\|_{2 p}, \quad X, Y \in \mathrm{L}^{2 p} .
$$

The Lyapunov's inequality:

$$
\|X\|_{p_{1}}=\left(\mathbb{E}\left[|X|^{p_{1}}\right]\right)^{1 / p_{1}} \leq\left(\mathbb{E}\left[|X|^{p_{2}}\right]\right)^{1 / p_{2}}=\|X\|_{p_{2}}, \quad 0<p_{1} \leq p_{2},
$$

implies $\mathrm{L}^{p_{2}} \subset \mathrm{L}^{p_{1}}$ and $p_{2}$-convergence implies $p_{1}$-convergence whenever $p_{1} \leq$ $p_{2}$.

Example 2. If $A$ is a r.v. such that

$$
\exists \alpha, M>0, \hat{t}>0: \quad\left\|t^{A-1}\right\|_{2} \leq M t^{\alpha-1}, \quad \forall t \geq \hat{t},
$$


then $\underset{t \rightarrow+\text { i.m. }}{\lim } e^{-t} t^{A-1}=0$. In fact, for $t \geq \hat{t}$,

$$
0 \leq\left\|e^{-t} t^{A-1}\right\|_{2}=e^{-t}\left\|t^{A-1}\right\|_{2} \leq M e^{-t} t^{\alpha-1} \underset{t \rightarrow+\infty}{\longrightarrow} 0 .
$$

Similarly, if $A$ is a r.v. such that

$$
\exists \alpha>1, M>0, \hat{t}>0: \quad\left\|t^{A-1}\right\|_{2} \leq M t^{\alpha-1}, \quad \forall 0<t \leq \hat{t},
$$

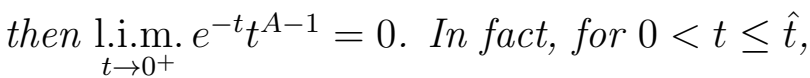

$$
0 \leq\left\|e^{-t} t^{A-1}\right\|_{2}=e^{-t}\left\|t^{A-1}\right\|_{2} \leq M e^{-t} t^{\alpha-1} \underset{t \rightarrow 0^{+}}{\longrightarrow} 0
$$

Note that, if an analogous condition to (5) [or to (6)] is fulfilled for a $p_{2}$ norm with $p_{2}>2$, then, by the Liapunov's inequality with $p_{1}=2$, condition (5) [or (6)] also holds. Later, the role of conditions (5) and (6) will be more apparent throughout our discussion.

Another result that will be used later is the Jensen inequality. Let $h$ be a convex function on $\mathbb{R}$. If $\mathbb{E}[|X|]$ and $\mathbb{E}[|h(X)|]$ are finite, then

$$
h(\mathbb{E}[X]) \leq \mathbb{E}[h(X)] .
$$

Observe that taking $h(x)=x^{2}$, this results guarantees that the variance $\mathbb{V}[X]=\mathbb{E}\left[X^{2}\right]-(\mathbb{E}[X])^{2}$ is always non-negative.

As we are interested in extending the gamma deterministic function to the m.s. random context and the gamma function is defined by the improper Riemann integral (1), next we remember the definition of m.s. Riemann integral of a 2-s.p. on a finite interval and, on the basis of this definition, we will introduce the improper m.s. Riemann integral of a 2-s.p. Characterizations of both types of m.s. Riemann integrals will also be given. All these definitions and results can be found in [13, pp. 99-101].

Definition 2. Let $[a, b]$ be an interval on the real line, and let $a=t_{n, 0}<$ $t_{n, 1}<\ldots<t_{n, n}=b$ be a partition such that $\Delta_{n}=\max \left\{t_{n, k}-t_{n, k-1}: k=1, \ldots, n\right\} \rightarrow$ 0 as $n \rightarrow+\infty$. Let $t_{n, k}^{\prime}$ be an arbitrary point in the interval $\left[t_{n, k-1}, t_{n, k}\right)$. Let $f(t, u)$ be a deterministic function defined on the same interval for the variable $t$ and Riemann integrable for every $u \in U$, where $U$ is a subset of $\mathbb{R}$. We say that the 2-s.p. $\{f(t, u) X(t): t \in T\}$ is mean square Riemann integrable 
over $[a, b]$ if for each $u \in U$ the following m.s. limit exists for some sequence of partitions of $[a, b]$

$$
\sum_{k=1}^{n} f\left(t_{n, k}^{\prime}, u\right) X\left(t_{n, k}^{\prime}\right)\left(t_{n, k}-t_{n, k-1}\right) \underset{n \rightarrow+\infty}{\stackrel{m . s .}{\longrightarrow}} Y(u),
$$

and in this case it is denoted by

$$
Y(u)=\int_{a}^{b} f(t, u) X(t) \mathrm{d} t
$$

This value is independent of the sequence of partitions as well as the position of $t_{n, k}^{\prime} \in\left[t_{n, k-1}, t_{n, k}\right)$. Note that, for each $u$, the integral is a random variable, since we integrate with respect to time.

The following result, that will be used later, characterizes the existence of the m.s. Riemann integral of a 2-s.p. in terms of the deterministic Riemann integral of its covariance function

Proposition 1. (see Theorem 4.5.1 of [13, p.100]) Let the s.p.

$$
Y(u)=\int_{a}^{b} f(t, u) X(t) \mathrm{d} t
$$

where $f(t, u)$ is a deterministic function $t$-Riemann integrable on $[a, b[$ for every $u \in U \subset \mathbb{R}$ and $\{X(t): a \leq t \leq b\}$ is a 2-s.p. with covariance function $\Gamma_{X}(t, s)$. Then, the s.p. $\{Y(u): u \in U\}$ exists if and only if the ordinary double Riemann integral

$$
\int_{a}^{b} \int_{a}^{b} f(t, u) f(s, u) \Gamma_{X}(t, s) \mathrm{d} t \mathrm{~d} s
$$

exists and is finite.

Improper m.s. Riemann integrals are defined in the same manner. In our case, the integrals will be improper of the first kind because $b=+\infty$ and, sometimes, when the integrand has a singularity at $t=a$, also improper of the second kind. So, we define

$$
\int_{a^{+}}^{\infty} f(t, u) X(t) \mathrm{d} t=\underset{\varepsilon \rightarrow 0^{+}, T \rightarrow \infty}{\lim .} \int_{a+\varepsilon}^{T} f(t, u) X(t) \mathrm{d} t .
$$


One can prove that this improper m.s. Riemann integral exists if and only if the improper ordinary double Riemann integral

$$
\int_{a^{+}}^{\infty} \int_{a^{+}}^{\infty} f(t, u) f(s, u) \Gamma_{X}(t, s) \mathrm{d} t \mathrm{~d} s
$$

exists and is finite (see [13, pp. 100-101]).

Remark 1. The existence and convergence of integral (11) can be guaranteed in terms of a unidimensional integral. In fact, by Jensen (see (7) with $h(x)=$ $|x|)$ and Schwarz's inequalities one gets:

$$
\left|\Gamma_{X}(t, s)\right|=|\mathbb{E}[X(t) X(s)]| \leq \mathbb{E}[|X(t) X(s)|] \leq\|X(t)\|_{2}\|X(s)\|_{2} .
$$

Then by the Fubini theorem

$$
\begin{gathered}
\int_{a^{+}}^{\infty} \int_{a^{+}}^{\infty}\left|f(t, u) f(s, u) \Gamma_{X}(t, s)\right| \mathrm{d} t \mathrm{~d} s \\
\leq \int_{a^{+}}^{\infty} \int_{a^{+}}^{\infty}|f(t, u)||f(s, u)|\|X(t)\|_{2}\|X(s)\|_{2} \mathrm{~d} t \mathrm{~d} s \\
=\left(\int_{a^{+}}^{\infty}|f(t, u)|\|X(t)\|_{2} \mathrm{~d} t\right)\left(\int_{a^{+}}^{\infty}|f(s, u)|\|X(s)\|_{2} \mathrm{~d} s\right) .
\end{gathered}
$$

Summarizing, if $f(t, u)$ is a deterministic function t-Riemann integrable on ] a, $\infty[$ for every $u \in U \subset \mathbb{R},\{X(t): t>a\}$ is a 2-s.p and the following deterministic integral

$$
\int_{a^{+}}^{\infty}|f(t, u)|\|X(t)\|_{2} \mathrm{~d} t
$$

is finite, then the stochastic integral $\int_{a^{+}}^{\infty} f(t, u) X(t) \mathrm{d} t$ is well-defined in the m.s. sense.

Although we are interested in establishing the random gamma function in the m.s. sense, as it was pointed out in the introductory section, throughout this paper we will need to use $\mathrm{L}^{p}$ stochastic calculus for $p=2$ and $p=4$. In fact, for instance the following $\mathrm{L}^{2}$-operational basic property

$$
\left.\begin{array}{c}
A \in \mathrm{L}^{2}, \\
\left\{X_{n}: n \geq 0\right\} \subset \mathrm{L}^{2}: X_{n} \underset{n \rightarrow \infty}{\stackrel{\text { m.s. }}{\longrightarrow}} X \in \mathrm{L}^{2},
\end{array}\right\} \Rightarrow A X_{n} \underset{n \rightarrow \infty}{\stackrel{\text { m.s. }}{\longrightarrow}} A X,
$$


does not hold, in general, as we will show in Examples 3 and 4. However, as it will be seen later, it can be established by including hypotheses involving $\mathrm{L}^{4}$ information.

Notice that, if $A$ and $\left\{X_{n}: n \geq 0\right\}$ are assumed to be independent for each $n$, then property (12) is fulfilled. Unfortunately, as we will see later, independence hypothesis cannot be embraced in our context. Nevertheless, the following result shows that the property (12) becomes true if we include appropriate $\mathrm{L}^{4}$ conditions in the hypotheses. From now on, the notation m.f. will stand for mean fourth convergence, i.e., convergence in the norm defined by (2) with $p=4$.

Lemma 1. Let $\left\{X_{n}: n \geq 0\right\}$ be a sequence in $\mathrm{L}^{4}$ such as $X_{n} \underset{n \rightarrow \infty}{\stackrel{m . f .}{\longrightarrow}} X$ and $A \in \mathrm{L}^{4}$. Then $A X_{n} \underset{n \rightarrow \infty}{\stackrel{m . s .}{\longrightarrow}} A X$.

Proof. The result follows straightforwardly by applying (4) for $p=2$ :

$$
\left\|A\left(X-X_{n}\right)\right\|_{2} \leq\|A\|_{4}\left\|X-X_{n}\right\|_{4} \underset{n \rightarrow \infty}{\longrightarrow} 0
$$

where, we have used that $\|A\|_{4}<\infty$ (since by hypothesis $A \in \mathrm{L}^{4}$ ) together with $\left\|X-X_{n}\right\|_{4} \underset{n \rightarrow \infty}{\longrightarrow} 0$ (since by hypothesis $X_{n} \underset{n \rightarrow \infty}{\stackrel{\text { m.f. }}{\longrightarrow}} X$ ).

The following couple of examples shows that the hypotheses assumed in Lemma 1 are minimum.

Example 3. ( $A \in \mathrm{L}^{2}$ is not sufficient). Let us consider a r.v. $A \in \mathrm{L}^{2}$ such that $\mathbb{E}\left[A^{4}\right]$ does not exist. Notice that if $A$ is a Pareto r.v., $A \sim \operatorname{Pa}(\alpha ; \beta)$, $\alpha \in \mathbb{R}, \beta>0$, then $\mathbb{E}\left[A^{m}\right]$ does not exist when $m \geq \alpha$. Hence, it is enough to take $A \sim \mathrm{Pa}(4 ; \beta)$. Let us define the sequence of r.v.'s

$$
X_{n}=\left\{\begin{array}{ccc}
0 & w . p . & 1-\frac{1}{n^{3}}, \\
n A & \text { w.p. } & \frac{1}{n^{3}},
\end{array}\right.
$$

(w.p. stands for with probability). Let us prove that $X_{n} \underset{n \rightarrow \infty}{\stackrel{m . s .}{\longrightarrow}} X=0$. For it, note that by the definition of conditional expectation one gets

$$
\mathbb{E}\left[\left(X_{n}-X\right)^{2} \mid A\right]=\mathbb{E}\left[\left(X_{n}\right)^{2} \mid A\right]=n^{2} A^{2} \frac{1}{n^{3}}=\frac{1}{n} A^{2}
$$


and applying conditional expectation properties one obtains

$$
\mathbb{E}\left[\left(X_{n}-X\right)^{2}\right]=\mathbb{E}\left[\mathbb{E}\left[\left(X_{n}-X\right)^{2} \mid A\right]\right]=\mathbb{E}\left[\frac{1}{n} A^{2}\right]=\frac{1}{n} \mathbb{E}\left[A^{2}\right] \underset{n \rightarrow \infty}{\longrightarrow} 0,
$$

since $\mathbb{E}\left[A^{2}\right]<\infty$. This proves that $X_{n} \underset{n \rightarrow \infty}{\stackrel{m . s .}{\longrightarrow}} X=0$. On the other hand, note that

$$
\left(A X_{n}\right)^{2}=\left\{\begin{array}{ccc}
0 & \text { w.p. } & 1-\frac{1}{n^{3}}, \\
n^{2} A^{4} & \text { w.p. } & \frac{1}{n^{3}},
\end{array}\right.
$$

Then

$$
\mathbb{E}\left[\left(A X_{n}\right)^{2}\right]=\mathbb{E}\left[\mathbb{E}\left[\left(A X_{n}\right)^{2} \mid A\right]\right]=\mathbb{E}\left[n^{2} A^{4} \frac{1}{n^{3}}\right]=\frac{1}{n} \mathbb{E}\left[A^{4}\right] .
$$

$A s \mathbb{E}\left[A^{4}\right]$ does not exist, the sequence $\left\{A X_{n}: n \geq 0\right\}$ does not converge in the m.s. sense to the null r.v. $X=0$.

Example 4. ( $X_{n} \underset{n \rightarrow \infty}{\stackrel{\text { m.s. }}{\longrightarrow}} X$ is not sufficient). Let $A$ be a Pareto r.v., $A \sim$ $\mathrm{Pa}(\alpha ; \beta)$ with $4<\alpha \leq 6$ and $\beta>0$. Let us consider the sequence of r.v.'s

$$
X_{n}=\frac{1}{n} A^{2}, \quad n \geq 0 .
$$

As $A \in \mathrm{L}^{4}, \mathbb{E}\left[A^{4}\right]<+\infty$ and

$$
\mathbb{E}\left[\left(X_{n}\right)^{2}\right]=\frac{1}{n^{2}} \mathbb{E}\left[A^{4}\right] \underset{n \rightarrow \infty}{\longrightarrow} 0 .
$$

Then, $X_{n} \underset{n \rightarrow \infty}{\stackrel{m . s .}{\longrightarrow}} X=0$. However, it does not converge to the null r.v., $X=0$, in the m.f. sense since

$$
\mathbb{E}\left[\left(X_{n}\right)^{4}\right]=\frac{1}{n^{4}} \mathbb{E}\left[A^{8}\right]
$$

and $\mathbb{E}\left[A^{8}\right]$ does not exist. On the other hand, as

$$
\mathbb{E}\left[\left(A X_{n}\right)^{2}\right]=\frac{1}{n^{2}} \mathbb{E}\left[A^{6}\right],
$$

$A X_{n}$ does not m.s. converge to $A X=0$ because $\mathbb{E}\left[A^{6}\right]$ does not exist. 
Remark 2. According to the previous exposition, as the m.s. Riemann integral of a 2-s.p. $X(t)$ is the m.s. limit of the Riemann sums of $X(t)$ (see [13, p.100]), the following basic property

$$
\int_{a}^{b} A X(t) \mathrm{d} t=A \int_{a}^{b} X(t) \mathrm{d} t,
$$

does not hold, in general, if $A$ and $X(t)$ are assumed to be in $\mathrm{L}^{2}$. Lemma 1 allows us to apply such property by assuming that $A \in \mathrm{L}^{4}$ and the Riemann integral of the 4-s.p. $X(t)$ is considered in the m.f. sense. Notice that, if the m.f. Riemann integral of the 4-s.p. $X(t)$ exists, then its m.s. Riemann integral also exists and coincides with the m.f. integral (see [15]).

Later, we will need to compute the m.s. derivative of a s.p. of the form $X(t)=f(Y(t))$, being $f$ a $\mathcal{C}^{1}(\mathbb{R})$ real-valued deterministic function and $Y(t)$ a 4-s.p. The following stochastic rule will be used to justify such computations:

Theorem 3 (Chain Rule). (see [15]) Let $f$ be a real-valued function with continuous derivative $f^{\prime}$, i.e. $f \in \mathcal{C}^{1}(\mathbb{R})$ and $\{Y(t): t>0\}$ be a 4-s.p. satisfying:

1. $Y(t)$ is m.f. differentiable.

2. $Y(t)$ is path continuous w.p. 1 .

3. There exist $r>4$ and $\delta>0$ such that $\sup _{s \in[-\delta, \delta]} \mathbb{E}\left[\left.\left|f^{\prime}(y)\right|_{y=Y(t+s)}\right|^{r}\right]<$ $+\infty$

Then, the 2-s.p. $X(t)=f(Y(t))$ is m.s. differentiable and its m.s. derivative is given by

$$
\frac{d X(t)}{d t}=\left.f^{\prime}(y)\right|_{y=Y(t)} \frac{d Y(t)}{d t} .
$$

Example 5. Let $X(t)=\left\{t^{A}: t>0\right\}$ be a s.p. where $A \in \mathrm{L}^{4}$. Then, by applying Theorem 3, let us justify that its m.s. derivative is: $\frac{d X(t)}{d t}=A t^{A-1}$. For this, we write $X(t)$ in the form $X(t)=f(Y(t))$, with $f(y)=e^{y}$ and $Y(t)=A \ln (t)$. Notice that $f(y) \in \mathcal{C}^{1}(\mathbb{R})$ and, by Example 1, $Y(t)$ is a 4-s.p. m.f. differentiable for $t>0$, being its m.f. derivative $\frac{d Y(t)}{d t}=\frac{A}{t}$. In addition, as $\ln (t)$ is continuous on $] 0, \infty[$, the s.p. $Y(t)$ is path continuous. If there exists $r>4$ and $\delta>0$ such that:

$$
\sup _{s \in[-\delta, \delta]} \mathbb{E}\left[(t+s)^{r A}\right]<+\infty
$$


then by (14): $\frac{d X(t)}{d t}=\left.e^{y}\right|_{y=A \ln (t)} \frac{A}{t}=t^{A} \frac{A}{t}=A t^{A-1}$, where this derivative is considered in the m.s. sense.

Remark 4. In Theorem 3, when $f(y)=e^{y}$, such as it happens in the previous example, condition 3 might, in some cases, be easier to verify if we check that, for some $\delta>0$,

$$
\sup _{s \in[t-\delta, t+\delta]} \sum_{n \geq 0} \frac{\left(\mathbb{E}\left[(Y(s))^{6 n}\right]\right)^{1 / 6}}{n !}<+\infty
$$

in which case the exponential s.p. defined as $e^{Y(t)}=\sum_{n \geq 0} \frac{(Y(t))^{n}}{n !}$ is in $\mathrm{L}^{6}$, [15]. This implies that $\mathbb{E}\left[\left(e^{Y(s)}\right)^{6}\right]$ is finite continuous for all $s \in[t-\delta, t+\delta]$ and condition 3 follows.

The following lemma will be used later to prove that a stochastic integral is well-defined in both m.s. and m.f. senses.

Lemma 2. (see Lemma 5 of [16]) Let $f$ be a real continuous function and $\{Y(x, t): x, t \in[a, b]\}$ a q-s.p. satisfying:

i) $Y(x, t)$ is path continuous on $[a, b] \times[a, b]$ w.p.1.

ii) There exist $r>q \geq 1$ and $\delta>0$ such that:

$$
\sup _{s, s^{*} \in[-\delta, \delta]} \mathbb{E}\left[\left.|f(y)|_{y=Y\left(x+s, t+s^{*}\right)}\right|^{r}\right]<\infty .
$$

Then, the q-s.p. $f(Y(x, t))$ is q-mean continuous at $(x, t)$.

To close this section, we point out that a good account of the relationship between $\mathrm{L}^{2}$ and $\mathrm{L}^{4}$ stochastic calculus can be found in [15].

\section{Introducing the gamma function for positive random variables}

The purpose of this section is to define, in the m.s. sense, the gamma function for positive r.v.'s $A$ and to determine conditions on $A$ for its existence. So, throughout this section, we assume that $A>0$ with probability one (w.p.1). 
Motivated by its deterministic definition (see expression (1)), it is natural to define $\Gamma(A)$ as the r.v.

$$
\Gamma(A)=\int_{0^{+}}^{\infty} e^{-t} t^{A-1} \mathrm{~d} t
$$

whenever this m.s. Riemann integral converges. As the argument is denoted by an upper case letter in the random scenario, there is no confusion between the deterministic $(\Gamma(\alpha))$ and random $(\Gamma(A))$ notation of the gamma functions.

From Remark 1, if $A$ is a r.v. such as $X(t)=t^{A-1}$ is a 2-s.p. and the following deterministic integral

$$
\int_{0^{+}}^{\infty} e^{-t}\left\|t^{A-1}\right\|_{2} \mathrm{~d} t
$$

is convergent, then the random gamma function (16) is well-defined in the m.s. sense.

The following example shows that $X(t)=t^{A-1}$ may not be a 2-s.p. even for standard positive 2-r.v.'s $A$.

Example 6. Let $A$ be an exponential r.v. of parameter $\lambda>0: A \sim \operatorname{Exp}(\lambda)$. Notice that $A$ is a positive r.v., i.e. $\mathbb{P}(\{\omega \in \Omega: A(\omega)>0\})=1$. Then,

$$
\left(\left\|t^{A-1}\right\|_{2}\right)^{2}=\frac{\lambda}{t^{2}} \int_{0^{+}}^{\infty} t^{2 a} e^{-\lambda a} \mathrm{~d} a=\left\{\begin{array}{cc}
\frac{\lambda}{t^{2}(\lambda-2 \ln (t))} & \text { if } t<e^{\lambda / 2}, \\
+\infty & \text { otherwise }
\end{array}\right.
$$

This means that $X(t)=t^{A-1}$ is not a 2-s.p. for all $t>0$ as it is required to give a satisfactory m.s. definition of the complete random gamma function that extends its deterministic counterpart.

The previous example motivates the search of conditions for the r.v. $A$ under which the deterministic integral (17) converges in order to guarantee that the stochastic integral (16) is well-defined in the m.s. sense.

Example 7. We will show that the set of s.p.'s $X(t)=t^{A-1}$ for which the random gamma function (16) is well-defined in the m.s. sense, is not empty. Let $A$ be a uniform r.v. on the interval $[2,3]: A \sim \mathrm{U}([2,3])$. First, we prove that $X(t)$ is a 2-s.p. for all $t>0$,

$$
n(t)=\left(\left\|t^{A-1}\right\|_{2}\right)^{2}=\mathbb{E}\left[t^{2 A-2}\right]=\int_{2}^{3} t^{2 a-2} \mathrm{~d} a=\frac{\left(t^{2}-1\right) t^{2}}{2 \ln t}<\infty, \quad \forall t>0 .
$$


Note that $n(1)=1$ and by L'Hôpital rule one gets

$$
\lim _{t \rightarrow 1} \frac{\left(t^{2}-1\right) t^{2}}{2 \ln t}=1
$$

Now, we need to check that (17) is convergent for $A \sim \mathrm{U}([2,3])$. To do that, we will need the following inequality

$$
\ln t \geq 1-\frac{1}{t}, \quad \forall t>0 .
$$

To show it, let us put $u(t)=\ln t+\frac{1}{t}-1$. For $0<t<1$, we have $\lim _{t \rightarrow 0^{+}} u(t)=$ $+\infty$ (easy to see by noting, using L'Hôpital rule, that $t u(t) \rightarrow 1$ when $t \rightarrow$ $\left.0^{+}\right), \lim _{t \rightarrow 1^{-}} u(t)=0$ and $u$ is decreasing since $u^{\prime}(t)=\frac{t-1}{t^{2}}<0$; therefore, $u(t) \geq 0$ and $(20)$ holds. For $t \geq 1$, we have $\lim _{t \rightarrow 1^{+}} u(t)=0$ and $u$ is non-decreasing since $u^{\prime}(t) \geq 0$; therefore, $u(t) \geq 0$ and (20) holds.

Let

$$
p(t)=\frac{t^{2}-1}{2 \ln t} .
$$

Using (20), one gets for $t>1$

$$
p(t)=\frac{t^{2}-1}{2 \ln t} \leq \frac{t^{2}-1}{2\left(1-\frac{1}{t}\right)}=\frac{t(t+1)}{2} \leq t^{2}, \quad t>1 .
$$

For $0<t<1$, one gets $\lim _{t \rightarrow 0^{+}} p(t)=0$, $\lim _{t \rightarrow 1^{-}} p(t)=1$ and $p(t)$ is increasing since

$$
p^{\prime}(t)=\frac{1-t^{2}+2 t^{2} \ln t}{2 t(\ln t)^{2}} \geq \frac{1-t^{2}+2 t^{2}\left(1-\frac{1}{t}\right)}{2 t(\ln t)^{2}}=\frac{(1-t)^{2}}{2 t(\ln t)^{2}}>0, \quad 0<t<1 ;
$$

therefore

$$
0<p(t)<1, \quad 0<t<1 .
$$

Then, taking into account (19) and inequalities (21)-(22) one gets

$$
\begin{aligned}
\int_{0^{+}}^{\infty} e^{-t}\left\|t^{A-1}\right\|_{2} \mathrm{~d} t & =\int_{0^{+}}^{\infty} e^{-t}\left(\frac{t^{2}-1}{2 \ln t} t^{2}\right)^{1 / 2} \mathrm{~d} t \\
& \leq \int_{0^{+}}^{1} e^{-t}\left(t^{2}\right)^{1 / 2} \mathrm{~d} t+\int_{1}^{\infty} e^{-t}\left(t^{4}\right)^{1 / 2} \mathrm{~d} t \\
& =\int_{0^{+}}^{1} e^{-t} t \mathrm{~d} t+\int_{1}^{\infty} e^{-t} t^{2} \mathrm{~d} t<\infty .
\end{aligned}
$$


Motivated by the previous example, next we introduce a class of r.v.'s for which the random gamma function (16) is well-defined in the m.s. sense. Let us assume that $A$ is a positive r.v. such that

$$
\exists M_{1}, M_{2}, \alpha_{1}, \alpha_{2}>0: \begin{cases}\left\|t^{A-1}\right\|_{2} \leq M_{1} t^{\alpha_{1}-1}, & 0<t \leq \hat{t}, \\ \left\|t^{A-1}\right\|_{2} \leq M_{2} t^{\alpha_{2}-1}, & \hat{t} \leq t<\infty,\end{cases}
$$

for some $\hat{t}>0$; then

$$
\begin{aligned}
\int_{0^{+}}^{\infty} e^{-t}\left\|t^{A-1}\right\|_{2} \mathrm{~d} t & =\int_{0^{+}}^{\hat{t}} e^{-t}\left\|t^{A-1}\right\|_{2} \mathrm{~d} t+\int_{\hat{t}}^{\infty} e^{-t}\left\|t^{A-1}\right\|_{2} \mathrm{~d} t \\
& \leq M_{1} \int_{0^{+}}^{\hat{t}} e^{-t} t^{\alpha_{1}-1} \mathrm{~d} t+M_{2} \int_{\hat{t}}^{\infty} e^{-t} t^{\alpha_{2}-1} \mathrm{~d} t \\
& \leq M_{1} \int_{0^{+}}^{\infty} e^{-t} t^{\alpha_{1}-1} \mathrm{~d} t+M_{2} \int_{0^{+}}^{\infty} e^{-t} t^{\alpha_{2}-1} \mathrm{~d} t \\
& =M_{1} \Gamma\left(\alpha_{1}\right)+M_{2} \Gamma\left(\alpha_{2}\right)<\infty .
\end{aligned}
$$

The following example shows that the r.v. $A$ considered in Example 7 fulfils conditions (23).

Example 8. If $A \sim \mathrm{U}([2,3])$, then checking computations contained in the Example 7, it is easy to see that

$$
\left\|t^{A-1}\right\|_{2} \leq \begin{cases}t & \text { if } \quad 0<t \leq 1 \\ t^{2} & \text { if } t \geq 1\end{cases}
$$

Therefore, conditions (23) are satisfied for $\hat{t}=1, M_{1}=M_{2}=1, \alpha_{1}=2$ and $\alpha_{2}=3$.

Remark 5. The exponential moment of a r.v., say $X$, is defined as $\mathbb{E}\left[t^{X}\right]$. So, rewriting the 2-norm in terms of the expectation operator, the inequality $\left\|t^{A-1}\right\|_{2} \leq M_{2} t^{\alpha_{2}-1}, t \geq \hat{t}$ included in condition (23) can be read as the exponential moments of r.v. $2 A$ growing at most as $t^{2 \alpha_{2}}, \alpha_{2}>0$, or equivalently, using Landau's notation, $\left\|t^{A-1}\right\|_{2}=\mathcal{O}\left(t^{\alpha_{2}-1}\right)$. 
Remark 6. In the Example 6 we showed that, if $A \sim \operatorname{Exp}(\lambda), \lambda>0$, then $\left\|t^{A-1}\right\|_{2}$ does not exist for $t \geq e^{\lambda / 2}$. As a consequence, condition (23) does not hold. This motivates the subsequent search of a general treatment that allows us to define the random gamma function for a wide range of positive r.v.'s.

Let us consider the set of all positive r.v.'s $A$ bounded and bounded away from 0, i.e. satisfying w.p.1

$$
0<h_{1} \leq A(\omega) \leq h_{2}<+\infty, \quad \forall \omega \in \Omega
$$

Notice that the function

$$
h(x)=t^{x}=e^{x \ln (t)}, \quad x \in \mathbb{R}, t>0
$$

satisfies

$$
\left\{\begin{array}{cl}
0<t<1 & \Rightarrow h(x) \text { is decreasing } \forall x \in \mathbb{R}, \\
t>1 & \Rightarrow h(x) \text { is increasing } \forall x \in \mathbb{R},
\end{array}\right.
$$

since $h^{\prime}(x)=\ln (t) e^{x \ln (t)}$ is negative (positive) for $0<t<1(t>1)$. Then, for $0<t<1$, as $h(x)$ is decreasing, one gets w.p.1

$$
2\left(h_{1}-1\right) \leq 2(A-1) \Rightarrow h(2(A-1)) \leq h\left(2\left(h_{1}-1\right)\right) \Rightarrow t^{2(A-1)} \leq t^{2\left(h_{1}-1\right)}
$$

and, as a consequence,

$$
\left(\left\|t^{A-1}\right\|_{2}\right)^{2}=\mathbb{E}\left[t^{2(A-1)}\right] \leq t^{2\left(h_{1}-1\right)} \Rightarrow\left\|t^{A-1}\right\|_{2} \leq t^{h_{1}-1}<\infty .
$$

If $t>1$, taking into account that $h(x)$ is increasing, an analogous reasoning yields w.p.1

$$
2(A-1) \leq 2\left(h_{2}-1\right) \Rightarrow h(2(A-1)) \leq h\left(2\left(h_{2}-1\right)\right) \Rightarrow t^{2(A-1)} \leq t^{2\left(h_{2}-1\right)},
$$

and so

$$
\left(\left\|t^{A-1}\right\|_{2}\right)^{2}=\mathbb{E}\left[t^{2(A-1)}\right] \leq t^{2\left(h_{2}-1\right)} \Rightarrow\left\|t^{A-1}\right\|_{2} \leq t^{h_{2}-1}<\infty .
$$

Therefore, if $A$ is a positive r.v. satisfying (24), then condition (23) holds taking $M_{1}=M_{2}=1, \hat{t}=1, \alpha_{1}=h_{1}$ and $\alpha_{2}=h_{2}$.

Summarizing, the following result has been established: 
Theorem 7. If $A$ is a positive r.v. satisfying condition (23), then the random gamma function $\Gamma(A)$ is well-defined in the mean square sense. In particular, (23) holds for every positive r.v. A satisfying condition (24) w.p.1.

Remark 8. Note that if $A$ is a positive r.v. satisfying (24), or satisfying the first part of (23), the random lower incomplete gamma function, $\Gamma_{L}(A, s)$, defined by

$$
\Gamma_{L}(A, s)=\int_{0^{+}}^{s} e^{-t} t^{A-1} \mathrm{~d} t
$$

is well defined in the m.s. sense. Now, if $A$ is a positive r.v. satisfying (24), or satisfying the second part of (23), the random upper incomplete gamma function, $\Gamma_{U}(A, s)$, defined by

$$
\Gamma_{U}(A, s)=\int_{s}^{\infty} e^{-t} t^{A-1} \mathrm{~d} t,
$$

is well defined in the m.s. sense. These special functions play an important role in deterministic Numerical Analysis, (see, for instance, [17, ch.10]). So, it is expected that its random extension impacts random numerics.

\section{Extending the gamma function to real random variables. Main statistical properties}

So far, we have shown (Theorem 7 ) that the m.s. gamma function $\Gamma(A)$ given by (16) is well defined when $A$ is a positive r.v. satisfying condition (23); in particular, that happens when $A$ is a positive r.v. satisfying condition (24). The aim of this section is to extend $\Gamma(A)$ to real-valued r.v.'s $A$. Motivated by the deterministic approach, this extension will be based on the rigorous proof, in the m.s. sense, of the formula $\Gamma(A+1)=A \Gamma(A)$. This requires the application of the following integration by parts formula for improper m.s. Riemann integrals, which is a direct extension of the integration by parts formula for m.s. proper Riemann integrals (see [13, p.104]).

Proposition 2. (improper m.s. Riemann integration by parts rule) Let $f(t)$ be a deterministic function of class $\mathcal{C}^{1}$ for $t>a$ and $\{X(t): t>a\}$ be a 2-s.p. m.s. differentiable such that its m.s. derivative $X^{\prime}(t)$ is m.s. continuous. If three of the four following m.s. limits

$$
\begin{aligned}
& \underset{\varepsilon \rightarrow 0^{+}, T \rightarrow \infty}{\lim } \int_{a+\varepsilon}^{T} f(t) X^{\prime}(t) \mathrm{d} t, \quad \underset{\varepsilon \rightarrow 0^{+}, T \rightarrow \infty}{\lim .} \int_{a+\varepsilon}^{T} f^{\prime}(t) X(t) \mathrm{d} t, \\
& \underset{T \rightarrow \infty}{\lim } f(T) X(T), \quad \underset{\varepsilon \rightarrow a^{+}}{\lim } f(a+\varepsilon) X(a+\varepsilon)
\end{aligned}
$$


exist, then the remaining m.s. limit also exists and one gets:

$$
\int_{a^{+}}^{\infty} f^{\prime}(t) X(t) \mathrm{d} t=\underset{T \rightarrow \infty}{\operatorname{lij.m}} f(T) X(T)-\underset{\varepsilon \rightarrow 0^{+}}{\operatorname{li} .{ }^{\prime}} f(a+\varepsilon) X(a+\varepsilon)-\int_{a^{+}}^{\infty} f(t) X^{\prime}(t) \mathrm{d} t .
$$

Let us assume that $A$ is a positive $r$-r.v. with $r>4$ such that

$$
\sup _{s \in[-\delta, \delta]} \mathbb{E}\left[(t+s)^{r A}\right]<+\infty
$$

for some $\delta>0$. Of course, if $A$ satisfies (28), so does $A+1$ and the reciprocal is also true.

Let us further assume that $A$ satisfies condition (23) with parameters $M_{1}>0, \alpha_{1}>0, M_{2}>0, \alpha_{2}>0$, and $\hat{t}>0$. Then $A+1$ also satisfies condition (23) with parameters $M_{1}>0, \alpha_{1}+1>1, M_{2}>0, \alpha_{2}+1>1$, and $\hat{t}>0$. Then, by Theorem 7 , both $\Gamma(A)$ and $\Gamma(A+1)$ are well defined in the m.s. sense.

Under these assumptions, we will now establish the formula $\Gamma(A+1)=$ $A \Gamma(A)$.

We will first apply the integration by parts formula (27) given in Proposition 2 for $a=0, f(t)=-e^{-t}$, and $X(t)=t^{A}$.

Note that, due to $(28), X(t)$ is a 4-s.p. and, therefore, also a 2-s.p. Applying Lemma 2 with fixed $x$ to $f(y)=e^{y}, Y(t)=A \ln t$ and taking into account that condition (15) is guaranteed from hypothesis (28), we conclude that $X(t)$ is m.f. continuous. By Example $5, X(t)$ is m.s differentiable with m.s. derivative $A t^{A-1}$; since $t^{A}$ is m.f. continuous, such derivative is m.s. continuous.

So, all conditions for application of Proposition 2 are verified. Notice that, by Example 2 applied to $A+1$, we conclude that $f(T) X(T)=e^{-T} T^{A}$ will converge in m.s. to 0 as $T \rightarrow+\infty$ and, since $\alpha_{1}+1>1$ we also conclude that $f(a+\varepsilon) X(a+\varepsilon)=e^{-\varepsilon} \varepsilon^{A}$ will converge in m.s. to 0 as $\varepsilon \rightarrow 0^{+}$. Therefore, by $(27)$, we have

$$
\int_{0^{+}}^{\infty} e^{-t} t^{A} \mathrm{~d} t=\int_{0^{+}}^{\infty} e^{-t} A t^{A-1} \mathrm{~d} t .
$$

Since $X(t)$ and so $e^{-t} t^{A-1}$ are m.f. continuous, the integrals $\int_{\varepsilon}^{T} e^{-t} t^{A-1} \mathrm{~d} t$ are well-defined in the m.f. sense (and coincide with the corresponding m.s. sense integrals, [15]). So, by Remark 2, we have $\int_{\varepsilon}^{T} e^{-t} A t^{A-1} \mathrm{~d} t=$ $A \int_{\varepsilon}^{T} e^{-t} t^{A-1} \mathrm{~d} t$ in the m.s. sense. Taking m.s. limits as $\varepsilon \rightarrow 0^{+}$and 
$T \rightarrow+\infty$, we obtain $\int_{0^{+}}^{\infty} e^{-t} A t^{A-1} \mathrm{~d} t=A \int_{0^{+}}^{\infty} e^{-t} t^{A-1} \mathrm{~d} t$. Therefore, in the m.s. sense, we obtain

$$
\Gamma(A+1)=\int_{0^{+}}^{\infty} e^{-t} t^{A} \mathrm{~d} t=A \int_{0^{+}}^{\infty} e^{-t} A t^{A-1} \mathrm{~d} t=A \Gamma(A) .
$$

Summarizing, the following result has been established:

Proposition 3. Let $A$ be a positive r-r.v. with $r>4$ such that conditions (23) and (28) hold. Then, both $\Gamma(A)$ and $\Gamma(A+1)$ are well defined and

$$
\Gamma(A+1)=A \Gamma(A) .
$$

Remark 9. In particular, if $A$ is a positive r.v. satisfying condition (24) w.p.1, it automatically satisfies all the conditions of this Proposition.

As in the deterministic case, formula (30) permits the extension, in the m.s. sense, of the random gamma function. Should $\Gamma(A+1)$ be well-defined in the m.s. sense by (16), the idea is to use (30) to define $\Gamma(A)$ in cases in which it can not be defined by (16). This is particularly useful when $A$ is not a positive r.v., but $A+1$ is. Of course, the procedure can be iterated.

Assume $A$ is a real-valued $r$-r.v. with $r>4$ satisfying condition (28). Assume further that the support of $A$ is w.p. 1 a subset of $S=]-1,-\alpha[\cup] \alpha,+\infty[$ for some small $\alpha>0$. This means that the support of $A$ should not contain a small neighborhood of zero and that $A$ may take negative values $>-1$, although the case of $A$ being a positive r.v. is not excluded. We are not assuming that $A$ satisfies condition (23), and so, even in the particular case of $A$ being a positive r.v., the requirements for the use of (16) to define $\Gamma(A)$ in the m.s. sense could not be met. However, we will now assume that $A+1$ does satisfy condition (23). Since $A+1$ is a positive $r$-r.v. also satisfying (28), we know from Theorem 7 that $\Gamma(A+1)$ is well-defined in the m.s. sense by (16), namely $\Gamma(A+1)=\int_{0^{+}}^{\infty} e^{-t} t^{A+1-1} \mathrm{~d} t$. We can now define $\Gamma(A)$ in the m.s. sense by

$$
\Gamma(A):=\frac{\Gamma(A+1)}{A} .
$$

Indeed, $\Gamma(A) \in \mathrm{L}^{2}$ since

$$
\mathbb{E}\left[(\Gamma(A))^{2}\right]=\mathbb{E}\left[\frac{1}{A^{2}}(\Gamma(A+1))^{2}\right] \leq \frac{1}{\alpha^{2}} \mathbb{E}\left[(\Gamma(A+1))^{2}\right]<\infty .
$$


Notice that $A+1$ satisfying (23) is equivalent to $A$ satisfying the condition $\exists M_{1}>0, M_{2}>0, \alpha_{1}>-1, \alpha_{2}>-1: \begin{cases}\left\|t^{A-1}\right\|_{2} \leq M_{1} t^{\alpha_{1}-1}, & 0<t \leq \hat{t}, \\ \left\|t^{A-1}\right\|_{2} \leq M_{2} t^{\alpha_{2}-1}, & \hat{t} \leq t<\infty,\end{cases}$ for some $\hat{t}>0$.

Iterating $\Gamma(A+1)=A \Gamma(A)$, we can now define $\Gamma(A)$ in the m.s. sense using, with $n$ positive integer,

$$
\Gamma(A):=\frac{\Gamma(A+n)}{A(A+1) \cdots(A+n-1)}
$$

as long as $A+n$ satisfies condition (23) and the support of r.v. $A$ is w.p.1 a subset of $S=]-n,-(n-1)-\alpha[\cup]-(n-1)+\alpha,-(n-2)-\alpha[\cup$ $\ldots \cup]-2+\alpha,-1-\alpha[\cup]-1+\alpha,-\alpha[\cup] \alpha,+\infty[$ for some small $\alpha>0$. This means that $A$ is allowed (but not required) to take negative values $>-n$ but its support should exclude small neighborhoods of $0,-1, \ldots,-(n-1)$. Indeed, $\Gamma(A)$ is well-defined in the m.s., i.e. $\Gamma(A) \in \mathrm{L}^{2}$, since

$$
\begin{aligned}
\mathbb{E}\left[(\Gamma(A))^{2}\right] & =\mathbb{E}\left[\frac{1}{(A(A+1) \cdots(A+n-1))^{2}}(\Gamma(A+n))^{2}\right] \\
& \leq \frac{1}{\alpha^{2 n}} \mathbb{E}\left[(\Gamma(A+n))^{2}\right]<\infty .
\end{aligned}
$$

Notice that $A+n$ satisfying (23) is equivalent to $A$ satisfying the condition

$\exists M_{1}>0, M_{2}>0, \alpha_{1}>-n, \alpha_{2}>-n: \begin{cases}\left\|t^{A-1}\right\|_{2} \leq M_{1} t^{\alpha_{1}-1}, & 0<t \leq \hat{t}, \\ \left\|t^{A-1}\right\|_{2} \leq M_{2} t^{\alpha_{2}-1}, & \hat{t} \leq t<\infty,\end{cases}$

for some $\hat{t}>0$.

We can do the same for the particular case of $A+n$ being a positive r.v. satisfying condition (24) w.p.1 (bounded and bounded away from 0), in which case $\Gamma(A+n)$ is well defined in the m.s. sense by $\Gamma(A+n)=$ $\int_{0^{+}}^{\infty} e^{-t} t^{A+n-1} \mathrm{~d} t$. Of course, to sucessfully apply (32), $A$ now needs to be bounded and bounded away from $-n$ and its support should exclude small neigborhoods of $0,-1, \ldots,-(n-1)$. In conclusion, we can define $\Gamma(A)$ in the m.s. sense by using (32) if $A$ is a r.v. which support is w.p.1 a subset of $S=[-n+\alpha,-(n-1)-\alpha[\cup]-(n-1)+\alpha,-(n-2)-\alpha[$ $\cup \ldots \cup]-2+\alpha,-1-\alpha[\cup]-1+\alpha,-\alpha[\cup] \alpha, L]$ for a small $\alpha>0$ and a finite $L>0$ as large as desired. 


\section{Numerical examples and computing}

As $\Gamma(A)$ is a r.v., it is also interesting to provide its main statistical properties such as the expectation, $\mathbb{E}[\Gamma(A)]$, and the variance, $\mathbb{V}[\Gamma(A)]=$ $\mathbb{E}\left[(\Gamma(A))^{2}\right]-(\mathbb{E}[\Gamma(A)])^{2}$. Using properties of m.s. Riemann integral (see $[13$, p.104-105]), one gets:

$$
\begin{aligned}
\mathbb{E}[\Gamma(A)] & =\int_{0^{+}}^{\infty} e^{-t} \mathbb{E}\left[t^{A-1}\right] \mathrm{d} t \\
\mathbb{E}\left[(\Gamma(A))^{2}\right] & =\int_{0^{+}}^{\infty} \int_{0^{+}}^{\infty} e^{-(t+s)} \mathbb{E}\left[(t s)^{A-1}\right] \mathrm{d} t \mathrm{~d} s .
\end{aligned}
$$

In the next examples, we illustrate the computation of the expectation and variance of $\Gamma(A)$.

Example 9. Let $A$ be a uniform r.v. on the interval [1,2]. As it is an absolutely continuous positive r.v. whose support is bounded, then, according to Theorem 7, the r.v. $\Gamma(A)$ is well-defined in the m.s. sense. Moreover

$$
\mathbb{E}[\Gamma(A)]=\int_{0^{+}}^{\infty} e^{-t}\left(\int_{1}^{2} t^{a-1} \mathrm{~d} a\right) \mathrm{d} t=0.922746,
$$

and

$\mathbb{V}[\Gamma(A)]=\int_{0^{+}}^{\infty} \int_{0^{+}}^{\infty} e^{-(t+s)}\left(\int_{1}^{2}(t s)^{a-1} \mathrm{~d} a\right) \mathrm{d} t \mathrm{~d} s-(\mathbb{E}[\Gamma(A)])^{2}=0.00114156$.

Example 10. Let $A$ be an exponential r.v. with parameter $\lambda=3$ truncated to the interval $[1,10]$. Again, notice that $\Gamma(A)$ is well-defined in the m.s. sense. In this case:

$$
\mathbb{E}[\Gamma(A)]=\int_{0^{+}}^{\infty} e^{-t}\left(\int_{1}^{10} \hat{f}_{A}(a) t^{a-1} \mathrm{~d} a\right) \mathrm{d} t=0.946847
$$

and

$\mathbb{V}[\Gamma(A)]=\int_{0^{+}}^{\infty} \int_{0^{+}}^{\infty} e^{-(t+s)}\left(\int_{1}^{10} \hat{f}_{A}(a)(t s)^{a-1} \mathrm{~d} a\right) \mathrm{d} t \mathrm{~d} s-(\mathbb{E}[\Gamma(A)])^{2}=0.590667$,

where

$$
\hat{f}_{A}(a)=\left\{\begin{array}{cl}
\frac{e^{-3 a}}{\int_{1}^{10} e^{-3 a} \mathrm{~d} a} & \text { if } a \in[1,10] \\
0 & \text { otherwise }
\end{array}\right.
$$

is the density function associated with the truncated r.v. A. 
Example 11. Let $A$ be a r.v. with density function

$$
f_{A}(a)=\left\{\begin{array}{cl}
\frac{1}{2\left(b_{1}-a_{1}\right)} & \text { if } a \in\left[a_{1}, b_{1}\right], \\
\frac{1}{2\left(b_{2}-a_{2}\right)} & \text { if } a \in\left[a_{2}, b_{2}\right], \\
0 & \text { otherwise, }
\end{array}\right.
$$

where $a_{1}=-2.9, b_{1}=-2.1, a_{2}=1, b_{2}=2$. As $0.1 \leq A+3 \leq 5$, condition (24) holds for $A+3$. Moreover, the support of $A$ excludes small neighborhoods of 0 and the negative integers. Therefore, $\Gamma(A)$ is well-defined in the m.s. sense and can be defined by (32) with $n=3$ and so one gets

$$
\begin{aligned}
\mathbb{E}[\Gamma(A)] & =\int_{a_{1}}^{b_{1}} \frac{1}{2\left(b_{1}-a_{1}\right)} \frac{\Gamma(a+3)}{a(a+1)(a+2)} \mathrm{d} a+\int_{a_{2}}^{b_{2}} \frac{1}{2\left(b_{2}-a_{2}\right)} \frac{\Gamma(a+3)}{a(a+1)(a+2)} \mathrm{d} a \\
& =-0.253131,
\end{aligned}
$$

and

$$
\begin{aligned}
\mathbb{V}[\Gamma(A)] & =\int_{a_{1}}^{b_{1}} \frac{1}{2\left(b_{1}-a_{1}\right)}\left(\frac{\Gamma(a+3)}{a(a+1)(a+2)}\right)^{2} \mathrm{~d} a \\
& +\int_{a_{2}}^{b_{2}} \frac{1}{2\left(b_{2}-a_{2}\right)}\left(\frac{\Gamma(a+3)}{a(a+1)(a+2)}\right)^{2} \mathrm{~d} a-(\mathbb{E}[\Gamma(A)])^{2} \\
& =1.66481 .
\end{aligned}
$$

In the next example the random Gamma function is applied to define the random Bessel function for a uniform random variable.

Example 12. We define the random Bessel function, denoted by $J_{A}(t)$, for a random variable $A$ with uniform distribution on the interval $[1,2]$ as

$J_{A}(t)=\frac{X(t)}{2^{A} \Gamma(A+1)}, \quad$ with $\quad X(t)=t^{A}\left[1+\sum_{n=1}^{\infty} \frac{(-1)^{n} t^{2 n}}{4^{n} n ! \prod_{i=1}^{n}(A+i)}\right], t>0$.

The s.p. $X(t)$ is m.f. convergent, see [10, p.7] and $\mathbb{E}\left[\left(\frac{1}{2^{A} \Gamma(A+1)}\right)^{4}\right]<\infty$, then $\mathbb{E}\left[\left(J_{A}(t)\right)^{2}\right]<\infty$. Moreover, A satisfies condition (24), thus by Remark 9 it follows that $\Gamma(A+1)=A \Gamma(A)$. This last property implies that $J_{A}(t)$ can be written as

$$
J_{A}(t)=\sum_{n=0}^{\infty} \frac{t^{A}}{2^{A} \Gamma(n+A+1)} \frac{(-1)^{n}}{n ! 4^{n}} t^{2 n} .
$$


To compute approximations of the mean and variance of $J_{A}(t)$, we truncate the infinite sum (33) at $N$ and define $J_{A}^{N}(t)$ by

$$
J_{A}^{N}(t)=\sum_{n=0}^{N} \frac{t^{A}}{2^{A} \Gamma(n+A+1)} \frac{(-1)^{n}}{n ! 4^{n}} t^{2 n} .
$$

Using expression (34) the mean of $J_{A}^{N}(t)$, denoted by $\mathbb{E}\left[J_{A}^{N}(t)\right]$, is given by

$$
\begin{aligned}
\mathbb{E}\left[J_{A}^{N}(t)\right] & =\sum_{n=0}^{N} \mathbb{E}\left[\frac{t^{A}}{2^{A} \Gamma(n+A+1)}\right] \frac{(-1)^{n}}{n ! 4^{n}} t^{2 n} \\
& =\sum_{n=0}^{N}\left(\int_{1}^{2} \frac{t^{A}}{2^{A} \Gamma(n+A+1)} d A\right) \frac{(-1)^{n}}{n ! 4^{n}} t^{2 n}
\end{aligned}
$$

and the variance of $J_{A}^{N}(t)$, denoted by $\mathbb{V}\left[J_{A}^{N}(t)\right]$, is

$$
\begin{aligned}
\mathbb{V}\left[J_{A}^{N}(t)\right] & =\mathbb{E}\left[\left(J_{A}^{N}(t)\right)^{2}\right]-\left(\mathbb{E}\left[J_{A}^{N}(t)\right]\right)^{2} \\
& =\sum_{n=0}^{N} \mathbb{E}\left[\frac{t^{2 A}}{4^{A}(\Gamma(n+A+1))^{2}}\right] \frac{1}{(n !)^{2} 4^{2 n}} t^{4 n} \\
& +2 \sum_{m=1}^{N} \sum_{n=0}^{m-1} \mathbb{E}\left[\frac{t^{2 A}}{4^{A} \Gamma(n+A+1) \Gamma(m+A+1)}\right] \frac{(-1)^{m+n}}{m ! n ! 4^{n+m}} t^{2(n+m)} \\
& -\left(\mathbb{E}\left[J_{A}^{N}(t)\right]\right)^{2},
\end{aligned}
$$

where

$\mathbb{E}\left[\frac{t^{2 A}}{4^{A}(\Gamma(n+A+1))^{2}}\right]=\int_{1}^{2} \frac{t^{2 A}}{4^{A}(\Gamma(n+A+1))^{2}} \mathrm{~d} A$,

$\mathbb{E}\left[\frac{t^{2 A}}{4^{A} \Gamma(n+A+1) \Gamma(m+A+1)}\right]=\int_{1}^{2} \frac{t^{2 A}}{4^{A} \Gamma(n+A+1) \Gamma(m+A+1)} \mathrm{d} A$.

In Tables 1 and 2 we have collected the numerical approximations of the mean and variance of $J_{A}(t)$ for different values of $t$ and $N$. It is observed that for $N=10,20$ the numerical values of the mean and the variance matched, respectively. 
Table 1: Approximations of the mean of $J_{A}^{N}(t)$ using expression (35) for $N=3,5,10,20$.

\begin{tabular}{ccccc}
\hline$t$ & $\mathbb{E}\left[J_{A}^{3}(t)\right]$ & $\mathbb{E}\left[J_{A}^{5}(t)\right]$ & $\mathbb{E}\left[J_{A}^{10}(t)\right]$ & $\mathbb{E}\left[J_{A}^{20}(t)\right]$ \\
\hline 1.0 & 0.072954 & 0.072955 & 0.072955 & 0.072955 \\
2.0 & 0.237481 & 0.237644 & 0.237644 & 0.237644 \\
2.5 & 0.256552 & 0.257843 & 0.257845 & 0.257845 \\
3.5 & 0.106055 & 0.122285 & 0.122401 & 0.122401 \\
4.0 & 0.030294 & 0.045701 & 0.045985 & 0.045985 \\
\hline
\end{tabular}

Table 2: Approximations of the variance of $J_{A}^{N}(t)$ using expression (36) for $N=3,5,10,20$.

\begin{tabular}{ccccc}
\hline$t$ & $\mathbb{V}\left[J_{A}^{3}(t)\right]$ & $\mathbb{V}\left[J_{A}^{5}(t)\right]$ & $\mathbb{V}\left[J_{A}^{10}(t)\right]$ & $\mathbb{V}\left[J_{A}^{20}(t)\right]$ \\
\hline 1.0 & 0.008953 & 0.008953 & 0.008953 & 0.008953 \\
2.0 & 0.004658 & 0.004668 & 0.004668 & 0.004668 \\
2.5 & 0.000549 & 0.000565 & 0.000565 & 0.000565 \\
3.5 & 0.010953 & 0.009021 & 0.009005 & 0.009005 \\
4.0 & 0.024517 & 0.016219 & 0.016105 & 0.016105 \\
\hline
\end{tabular}

\section{Conclusions}

In this paper, a generalization to the random scenario of the gamma function has been made. To conduct this extension, first the gamma function, $\Gamma(A)$, has been introduced for a class of positive random variables $A$ satisfying certain conditions related to their exponential moments. This class includes the positive random variables that are bounded and bounded away from zero. Then, establishing the formula $\Gamma(A+1)=A \Gamma(A)$ in the random scenario, 
we have extended $\Gamma(A)$ to a class of random variables $A$ whose support lies over the real line with the exception of small neighborhoods of zero and of the negative integers. The study has been based on the application of the mean square and mean fourth stochastic calculus. The obtained results extend its deterministic counterpart. In a forthcoming work, we will take advantage of this approach to generalize to the random scenario other important special functions.

\section{Funding}

This work has been partially supported by the Spanish M.C.Y.T. grant: MTM2013-41765-P and Mexican Conacyt. Carlos A. Braumann is a member of the Centro de Investigação em Matemática e Aplicações (CIMA), Universidade de Évora, a research centre supported with Portuguese funds by FCT (Fundação para a Ciência e a Tecnologia, Portugal) through the Project $\mathrm{UID} / \mathrm{MAT} / 04674 / 2013$.

\section{Acknowledgements}

Authors gratefully acknowledge the comments made by reviewers, which have greatly enriched the manuscript.

\section{Conflict of Interest Statement}

The authors declare that there is no conflict of interests regarding the publication of this article.

\section{References}

[1] E. D. Rainville, Special Functions, MacMillan, New York, 1960.

[2] G. N. Watson, A Treatise on the Theory of Bessel Functions, Cambridge University Press, New York, 1995.

[3] A. Golmankhaneh, N. Porghoveh, D. Baleanu, Mean square solutions of second-order random differential equations by using homotopy analysis method, Romain Reports in Physics 65 (2) (2013) 350-362. 
[4] L. Shaikhet, Necessary and sufficient conditions of asymptotic mean square stability for stochastic linear difference equations, Applied Mathematics Letters 10 (3) (1997) 111-115. doi:10.1016/S08939659(97)00045-1.

[5] L. Villafuerte, B. Chen-Charpentier, A random differential transform method: Theory and applications, Applied Mathematics Letters 25 (10) (2012) 1490-1494. doi:10.1016/j.aml.2011.12.033.

[6] M. Khodabin, K. Maleknejad, M. Rostami, M. Nouri, Numerical solution of stochastic differential equations by second order Runge Kutta methods, Mathematical and Computer Modelling 59 (53) (2011) 19101920. doi:10.1016/j.mcm.2011.01.018.

[7] B. Paternoster, L. Shaikhet, Mean square summability of solution of stochastic difference second-kind Volterra equation with small nonlinearity, Advances in Difference Equations (2007) 2007-ID65012, 13 pagesdoi:10.1155/2007/65012.

[8] M. A. El-Tawil, M. El-Sohaly, Mean square numerical methods for initial value random differential equations, Open Journal of Discrete Mathematics 1 (1) (2011) 164-171. doi:10.4236/ojdm.2011.12009.

[9] K. N. N. V. Lupulescu, Random fractional differential equations, International Electronic Journal of Pure and Applied Mathematics 4 (2) (2012) 119-136.

[10] J. C. Cortés, L. Jódar, L. Villafuerte, Mean square solution of Bessel differential equation with uncertainties, J. Computational Applied Mathematics 309 (2017) 383-395. doi:10.1016/j.cam.2016.01.034.

[11] L. Arnold, Stochastic Differential Equations: Theory and Applications, John Wiley \& Sons, New York, 1974.

[12] M. Loève, Probability Theory, Van Nostrand, Princeton, New Jersey, 1963.

[13] T. T. Soong, Random Differential Equations in Science and Engineering, Academic Press, New York, 1973. 
[14] E. Wong, H. B., Stochastic Processes in Engineering System, Springer Verlag, New York, 1985.

[15] L. Villafuerte, C. Braumann, J. C. Cortés, L. Jódar, Random differential operational calculus: Theory and applications, Computers and Mathematics with Applications 59 (1) (2010) 115-125. doi:10.1016/j.camwa.2009.08.061.

[16] J. C. Cortés, L. Jódar, M. D. Roselló, L. Villafuerte, Solving initial and two-point boundary value linear random differential equations: A mean square approach, Applied Mathematics and Computation 219 (4) (2012) 2204-2211. doi:10.1016/j.amc.2012.08.066.

[17] G. Arfken, H. Weber, Mathematical Methods for Physicists, Harcourt/Academic Press, 2000. 